\title{
CONTROLADORES PARA MOTORES DC E BRUSHLESS
}

Thiago Rodrigues, Henrique Ferreira Kazama, João Victor Pereira da Silva, César Daltoé Berci

Universidade do Oeste Paulista - UNOESTE, Curso de Engenharia Elétrica, Presidente Prudente, SP. E-mail: thiagorodrigues@unoeste.br.

\section{RESUMO}

O mercado de veículos com tração elétrica está sofrendo um grande impulso através de políticas nacionais e principalmente internacionais. Esse quadro poderá criar diversas demandas que hoje não existem, sendo uma delas o de acionamentos elétricos para motores automotivos. Nesse contexto, pretende-se desenvolver tecnologias de sistemas eletrônicos de acionamento e controle de motores, com capacidade para maximizar o aproveitamento em veículos elétricos, utilizando as propriedades dos semicondutores disponíveis atualmente no mercado. Este trabalho tem ainda o intuito de informar o leitor, sobre funcionamento de um motor brushless DC, a fim de esclarecer dúvidas sobre este tema.

Os controladores desenvolvidos serão então empregados no controle de motores para veículos elétricos, incluindo mecanismos de superação visando extrair potência acima da nominal em situações nas quais seja necessária a resposta rápida do motor.

\section{AND BRUSHLESS ENGINE CONTROLLERS}

\begin{abstract}
The electric-powered vehicles market has been driven by national and mainly international rules, what could create several demands that today still do not exist, one of them are the power drives for automotive electric motors. In this context, it is intended to develop electronic systems for drivers and motor controllers, with capacity to maximize its use in electric vehicles, by means of the optimal use of the properties of currently available semiconductors. This work intends to inform the reader about the operation of a brushless DC motor, in order to clarify doubts on this topic.

The developed controllers will then be used to control motors for electric vehicles, including overrun mechanisms to extract power above rated power in situations where fast engine response is required.
\end{abstract}




\section{INTRODUÇÃO}

O interesse por essa pesquisa veio com o recente aumento na demanda por veículos movidos por tração elétrica, o que se deu, parcialmente, em virtude de algumas desvantagens dos veículos a combustão com relação à econômica e eficiência do ponto de vista energético, mobilidade urbana e na sustentabilidade, principalmente no tangente à poluição ambiental, por utilizares motores considerados um dos principais poluidores atmosféricos. Com a popularização dos carros elétricos espera-se uma redução no nível de poluição gerado pela queima de combustíveis fósseis e um aumento na qualidade do ar, pois haverá menos veículos emitindo gases nocivos (ROMM, 2006).

O advento dos veículos com tração elétrica aumenta significativamente a demanda por dispositivos de controle de motores. Apesar da tecnologia desses dispositivos ser amplamente conhecida pela indústria, que possui atualmente diversos modelos de controladores comerciais para todos os tipos de motores, o desenvolvimento de novos componentes, com capacidade de corrente ampliada e características de comutação superiores, permitem o desenvolvimento de novos controladores mais eficientes e compactos (BLAABJERG; IONEL, 2015).

Os controladores desenvolvidos serão então empregados no controle de motores para veículos elétricos, incluindo mecanismos de superação, visando extrair potência acima da nominal em situações onde seja necessária uma resposta rápida do motor.

A pesquisa tem como objetivo desenvolver e criar protótipos de controladores de potência para motores DC e Brushless, tendo como objetivos específicos os seguintes pontos: Circuitos de potência para acionamento de motores DC utilizando modulação PWM; Circuitos de potência com conversores Buck para controle suave de motores DC, eliminando o ruído e a vibração; Circuitos de potência em ponte $\mathrm{H}$ para acionamento e reversão de motores $\mathrm{DC}$; Circuitos de potência para acionamento e controle de motores Brushless; Implementação de sistemas microprocessados e softwares embarcados para o acionamento e controle de motores DC e Brushless; Sistemas para controle, monitoramento e superação de motores.

Os objetivos aqui apresentados envolveram o desenvolvimento final do projeto, que foi dividido em três etapas sequenciais, a fim de organizar o processo de desenvolvimento geral de forma exequível.

\section{METODOLOGIA}

A primeira etapa foi na escolha dos dispositivos eletrônicos que foram usados como comutadores (chaves), para esse trabalho foram considerados transistores do tipo MOSFET ou IGBT. Essa escolha foi feita em função das características técnicas de ambos os componentes, tendo sido considerados: tempo de resposta de chaveamento; corrente máxima suportada e dissipação de calor. Esses requisitos foram fundamentais para escolha do dispositivo usado no protótipo atual do Drive de potência, que foi o IGBT.

O segundo componente a ser dimensionado foi o Gate Driver, utilizado para controlar o acionamento do dispositivo de chaveamento eletrônico. Esse componente tem por objetivo transformar os níveis de tensão e corrente do microcontrolador de forma a proporcionar o correto acionamento para os MOSFET's ou IGBT's. Para este trabalho foi utilizado o componente IR2101.

Por fim, definiu-se o microcontrolador a ser utilizado em função das necessidades de tempo e processamento do projeto. Nesse caso, o microcontrolador de 8 bits, PIC16F877A, fabricado pela Microchip foi escolhido em função de sua disponibilidade, arquitetura e periféricos.

Já na última etapa foram implementados os testes e simulações dos circuitos criados para o drive de potência. O protótipo foi então construído utilizando o modelo computacional por meio de um processo de fabricação assistida por computador utilizando fresagem CNC.

O software embarcado também foi desenvolvido e testado, tendo como finalidade implementar a leitura de um encoder incremental, utilizando código gray, e converter essa leitura 
em largura de pulso no sinal de PWM. Também foram implementadas rotinas básicas de proteção do motor.

\section{RESULTADOS}

Uma das variáveis a serem consideradas no dimensionamento do sistema é o tempo de resposta dos comutadores em função dos requisitos de tempo do sinal de PWM, o qual é função direta dos parâmetros do modelo dinâmico do motor (SINGH; KOHLI, 1984). Nos motores, o chaveamento provoca surtos de tensão em virtude de efeitos indutivos sob o circuito de acionamento. Esse fenômeno causa nos IGBT's, ao trabalhar com uma frequência muito alta, um expressivo aumento nas sobre tensões, que podem danificar os circuitos. Por outro lado, se a comutação for muito lenta, não será suficiente para acionar o motor de forma eficiente, assim por consequência o IGBT não será capaz de transmitir a potência necessária, caso não seja escolhida uma frequência compatível.

Utilizando a série de Fourier foi feita a análise do comportamento em frequência do sinal PWM, utilizando a seguinte equação (FIGUEIREDO, 1977).

$$
f(x)=\frac{a_{0}}{2}+\sum_{n=1}^{\infty} a_{n} \cos \left(\frac{n \pi t}{L}\right)+\sum_{n=1}^{\infty} b_{n} \sin \left(\frac{n \pi t}{L}\right)
$$

Através desta equação é possível identificar a amplitude harmônica das componentes do sinal PWM. A análise dessas amplitudes harmônicas é necessária, pois, tanto os IGBT's quanto os drivers de gate precisam capazes de reproduzi-las de tal forma a manter uma representação com o mínimo de fidelidade do sinal de PWM ideal.

Para obter o controle de PWM é preciso que o microcontrolador possua esse periférico (módulo PWM) em sua arquitetura ${ }^{1}$, essa saída gera um sinal cíclico e pulsado, controlado por software, sendo possível alterar os valores de frequência e largura de pulso, mas detalhes na referencia (BLAABJERG; NEACSU; PEDERSEN, 1999). Via de regra, o microcontrolador não consegue acionar diretamente os comutadores, em virtude dos níveis de tensão que disponibilizam em suas saídas digitais, por esse motivo é utilizado no circuito um componente de interface entre controle e potência, chamado GATE-DRIVE. Sua função é de ajustar os níveis de tensão e corrente de que o microcontrolador dispõe, afim de prover os requisitos necessários para a comutação dos dispositivos de acionamento, IGBT's e MOSFET's, que possuem alta capacitância no gate exigindo drivers tipo push-pull.

A utilização do gate driver, apesar de eliminar o problema de compatibilidade, requer um estudo de adequação, que incluí calcular tempo de resposta do GATE- DRIVE e verificar se este é adequado ao IGBT. Para fazer esse cálculo foram consultadas as folhas de dados dos fabricantes de ambos os dispositivos.

Tempos de resposta do IGBT (IRGP4066):

I. Tempo de acionamento do IGBT $T_{d(\text { on })}=50 \mathrm{~ns}$

II. Tempo de desligamento do IGBT $T_{d(\text { off })}=240 \mathrm{~ns}$

III. $\quad$ Rising time $T_{r}=$

IV. Rising time $T_{r}=70 n s$

V. Falling time $T_{f}=70 \mathrm{~ns}$

VI. Considerando como tempo mínimo de resposta: $T_{d(\text { on })}+T_{d(\text { off })}+T_{r}+T_{f}=$ $430 \mathrm{n}$, o que leva a uma frequência máxima de resposta nas condições especificadas de $2,33 \mathrm{MHz}$,

\footnotetext{
${ }^{1}$ Seria possível a utilização de um microprocessador sem módulo PWM, que seria então emulado por software ou implementado por hardware. Ambas as soluções se mostraram mais dispendiosas que utilizar um microprocessador com módulo PWM integrado.
} 
ou seja, o IGBT responde até a amplitude harmônica de ordem 4651 do controlador proposto, em virtude da frequência do sinal de PWM utilizado.

Tempo de reposta do GATE-DRIVE (IR2101)

I. Tempo de acionamento do IR2101T $T_{d(\text { on })}=130 \mathrm{~ns}$

II. Tempo de desligamento do IR2101 $T_{d(o f f)}=40 \mathrm{~ns}$

III. $\quad$ Rising time $T_{r}=80 \mathrm{~ns}$

IV. $\quad$ Falling time $T_{f}=40 \mathrm{~ns}$

Considerando como tempo mínimo de resposta do IGBT:

$T_{d(\text { on })}+T_{d(\text { off })}+T_{r}+T_{f}=430 n$,

Podemos calcular tempo mínimo para o IR2101:

$T_{d(\text { on })}+T_{d(\text { off })}+T_{r}+T_{f}=340$

Com os dois dados de tempo calculados, chegou-se a seguinte conclusão, o IGBT tem maior tempo de resposta devido a sua finalidade de aplicação ser voltada para comutação de cargas mais elevadas, assim, terá por característica esse tempo maior de comutação. Para o protótipo do Drive de Potência desenvolvido, o IGBT não será exigido em toda sua gama de resposta em frequência, de tal sorte que, utilizando apenas $10 \%$ desse valor total, por volta de $44 \mathrm{~ns}$, será suficiente para a aplicação proposta. Sendo assim, com o tempo de reposta obtido com o IR2101 pode-se trabalhar sem que haja nenhum problema na comutação final do motor. Com esses resultados foram determinadas duas das partes que formam o Drive de potência proposto para motores DC sem escovas: A interface entre controle e potência e os comutadores que irão fazer o chaveamento, restando o processamento e proteção do driver.

A proteção será realizada através de diodos de roda livre, instalados com a finalidade de suprimir surtos de tensão causados pela abertura do circuito do motor.

O processamento será feito pelo microcontrolador, que irá ler um sensor de posição incremental, tipo encoder (utilizando o código gray) e utilizará esse valor de leitura para ajustar os parâmetro do sinal de PWM aplicado a base dos dispositivos de chaveamento.

Tendo sido determinados todos os parâmetros, o modelo computacional do driver foi então desenvolvido e serviu como base para a fabricação da placa de circuito impresso conforme as figuras 1,2 e 3 . 
Figura 1. Diagrama eletrônico.

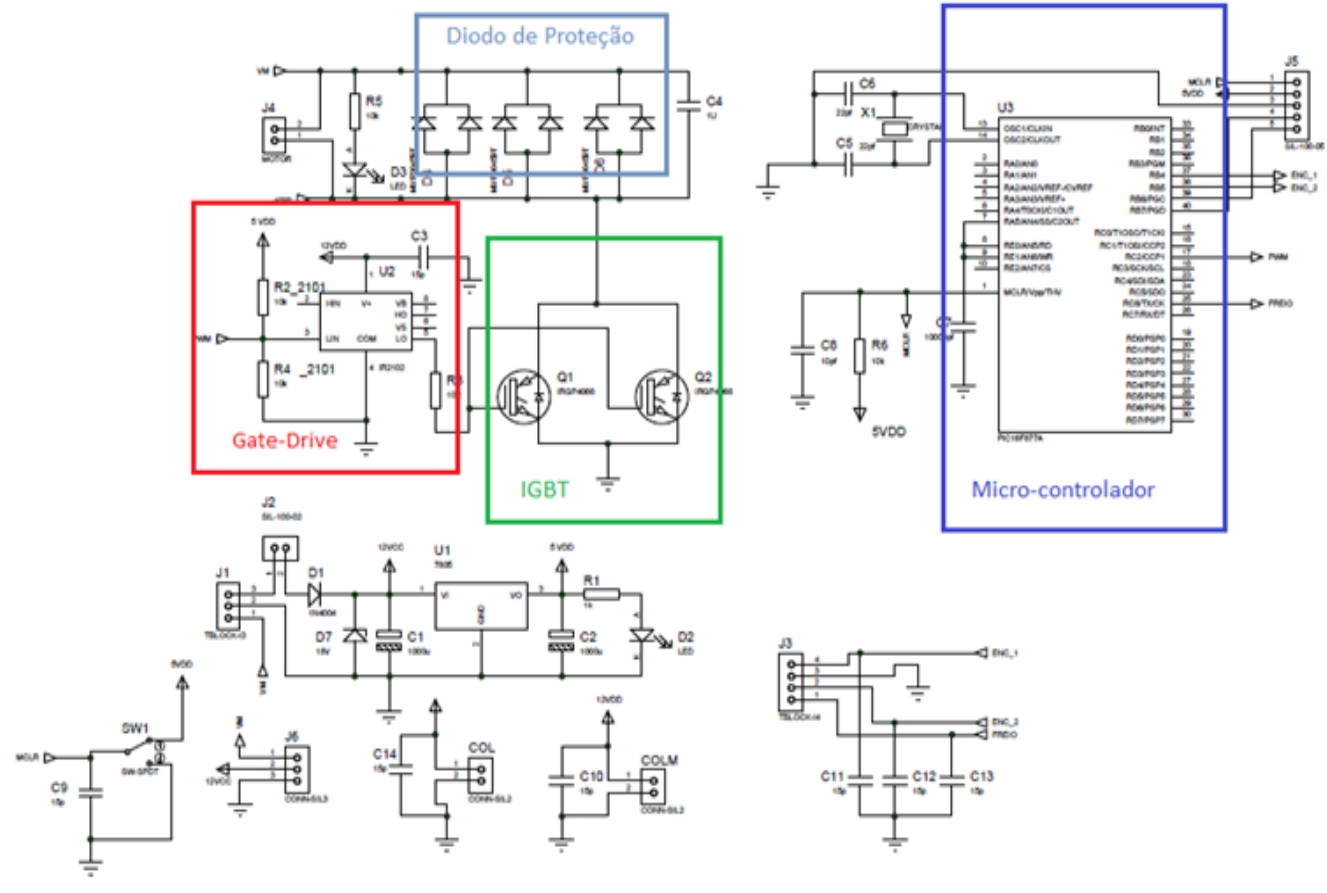

Fonte: autor 2017

Figura 2. Layout para placa de circuito

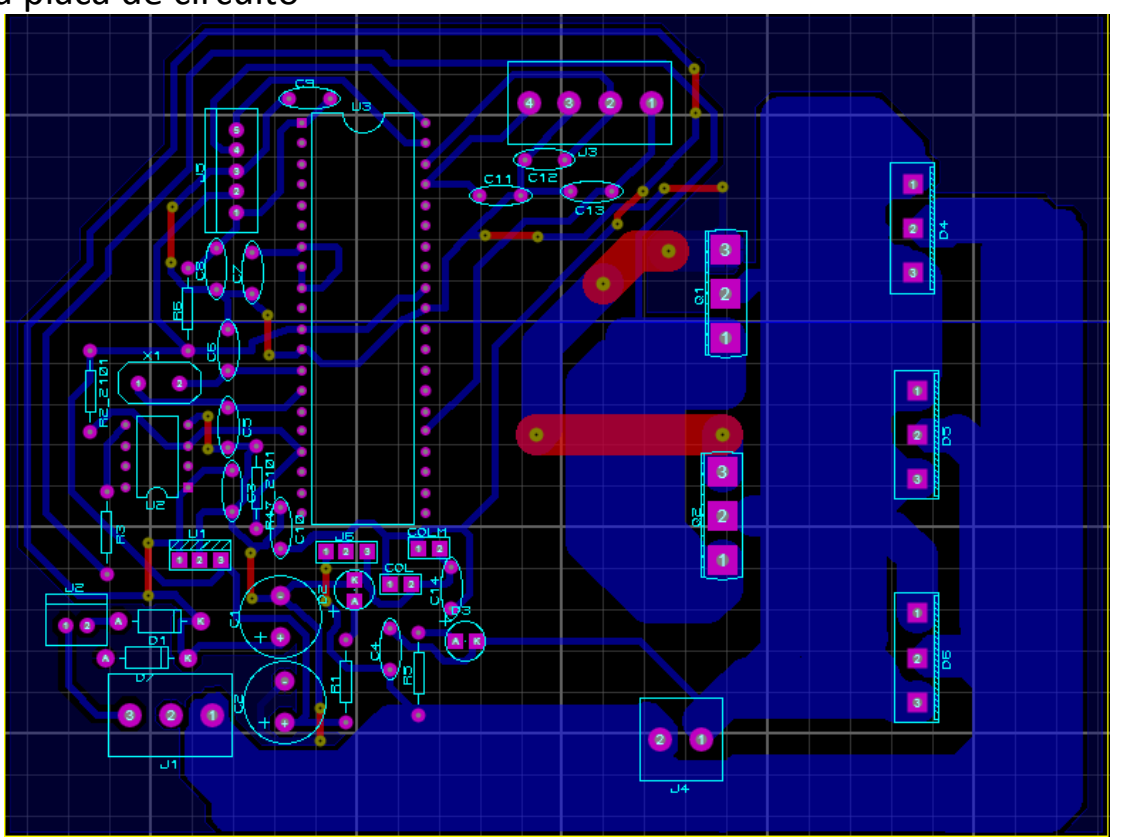

Fonte: autor 2017 
Figura 3. Representação 3D do drive .

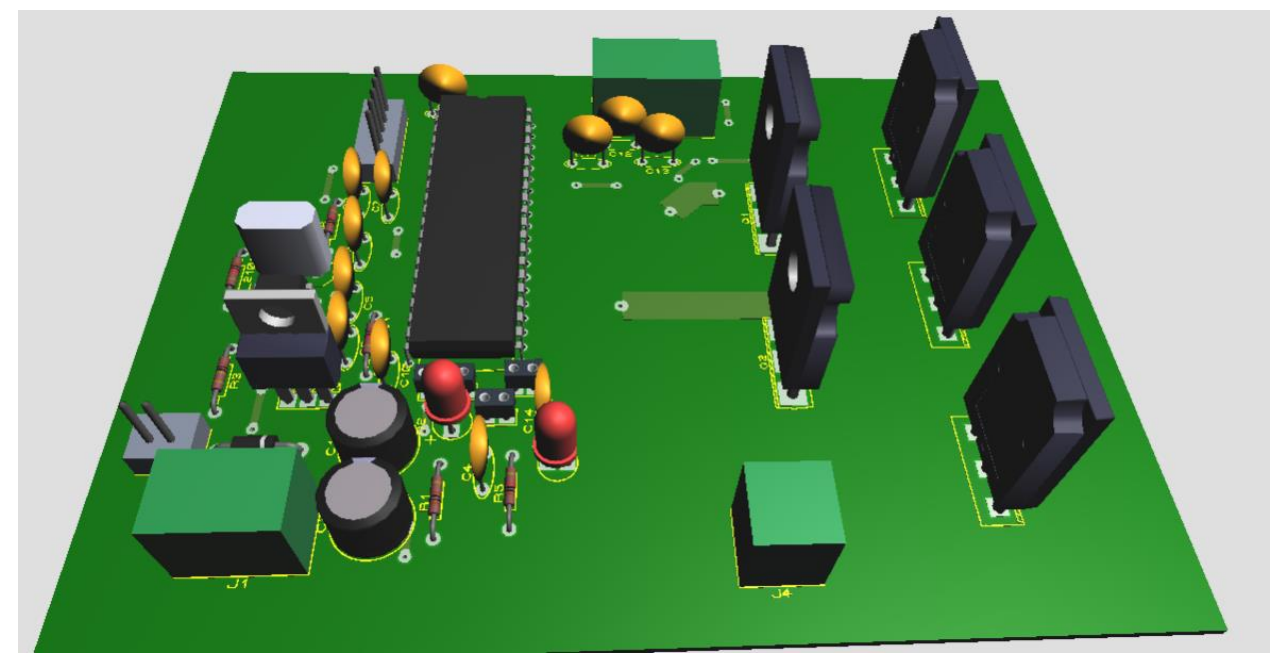

Fonte: autor 2017

\section{DISCUSSÃO}

O protótipo criado foi então instalado no veículo elétrico desenvolvido pelo curso de engenharia elétrica, para teste de funcionamento em campo.

Após dificuldades iniciais com a supressão dos surtos de tensão reversa, foi preciso adicionar mais diodos de proteção ao circuito, o que foi feito com base nas teorias de paralelismo de elementos de potência, pois, no momento da frenagem, principalmente, a força contra eletromotriz aliada a alta indutância do motor causam um surto de tensão superior ao esperado, tanto em amplitude quando em energia.

Esses diodos adicionais servem como uma barreira extra de proteção, os diodos utilizados foram do tipo MBR3045, escolhidos para esse modelo por suportar uma maior corrente de condução direta com baixo tempo polarização direta.

\section{CONCLUSÃO}

A pesquisa foi realizada pensando num futuro próximo, onde encontrará aplicação em muitos meios de transporte com tração elétrica. No Brasil já existem alguns veículos dessa categoria e a tendência é de crescimento a altas taxas desse número, segundo o site do Instituto Nacional de Eficiência Energética que se encontra na referencia (INEE, 2017).

Então, a ideia da pesquisa é desenvolver uma família de Drivers de Potência que sejam menos onerosos e apresentem boa eficiência, quando comparados ao que é disponível hoje no mercado, e que possa ser usado tanto em carros com tração elétrica quanto em veículos de tração hibrida, que unem motores elétricos e a combustão, tornando-se uma alternativa nacional que irá contribuir para viabilização da difusão desses veículos no mercado brasileiro.

Durante a pesquisa foi observado que é extremamente difícil encontrar componentes eletrônicos para essa aplicação, com mais tempo de estudo e com os resultados obtidos nesse trabalho, será possível aprimorar alguns parâmetros para modelagem de futuros novos dispositivos, evoluindo significativamente o protótipo atual.

O desafio da montagem do protótipo foi justamente utilizar os MOSFETS ou IGBT disponíveis, apoiando-se em teorias de paralelismo de componentes de potência, tornando o equipamento mais barato e acessível dentre os que se encontra hoje no mercado. 


\section{REFERÊNCIAS}

BLAABJERG, F.; IONEL, D. M. Renewable energy devices and systems-state-of-the-art technology, research and development, challenges and future trends. Electr Power Compon Syst 43(12):13191328, 2015, https://doi.org/10.1080/15325008.2015.1062819

BLAABJERG, F.; NEACSU, D. O.; PEDERSEN, J. K. Adaptive SVM to compensate dc-link voltage ripple for four-switch three-phase voltage-source inverter. IEEE Transactionson Power Electronics, Vol.14, pp. 743-752, 1999, https://doi.org/10.1109/63.774214

FIGUEIREDO, D. G. Análise de Fourier e equações diferenciais parciais. IMPA, Riu de Janeiro, 1977.

INEE. Site do Instituto Nacional de Eficiência Energética. Disponível em: <http://www.inee.org.br/>. Acesso em 17 jun. 2017.

ROMM, J. The car and fuel of the future. Energy Policy, Volume 34, Issue 17, November 2006, Pages 2609-2614, 2006.

SINGH, S. N.; KOHLI, D. R. Performance Determination of a Chopper-Controlled Separately Excited DC Motor in IEEE Transactions on Industrial Electronics, vol. IE-31, no. 1, pp. 37-42, Feb. 1984. doi: 10.1109/TIE.1984.350018, https://doi.org/10.1109/TIE.1984.350018. 